\title{
Bibnum
}

Textes fondateurs de la science

Sciences de la vie | 2017

\section{Karl Landsteiner (1868-1943) et la découverte des groupes sanguins}

Jean-Pierre Aymard

\section{(2) OpenEdition}

\section{Journals}

Édition électronique

URL : https://journals.openedition.org/bibnum/521

DOI : 10.4000/bibnum.521

ISSN : 2554-4470

\section{Éditeur}

FMSH - Fondation Maison des sciences de l'homme

\section{Référence électronique}

Jean-Pierre Aymard, «Karl Landsteiner (1868-1943) et la découverte des groupes sanguins », Bibnum [En ligne], Sciences de la vie, mis en ligne le 01 janvier 2012, consulté le 04 février 2023. URL : http:// journals.openedition.org/bibnum/521 ; DOI : https://doi.org/10.4000/bibnum.521 


\section{Karl Landsteiner (1868-1943) et la découverte des groupes sanguins}

par Jean-Pierre Aymard Docteur en Médecine, spécialiste en hématologie et transfusion sanguine Membre de la Société Française d'Histoire de la Médecine

La connaissance des groupes sanguins a progressé en lien étroit avec le développement de la transfusion sanguine. Mais la découverte des premiers d'entre eux, les groupes du système ABO, apparaît comme un des premiers succès de l'immunologie naissante : très antérieure à l'essor de la transfusion sanguine, elle est due à Karl Landsteiner et date de 1900-1901. Cette découverte lui vaudra le prix Nobel de Physiologie ou Médecine en 1930.

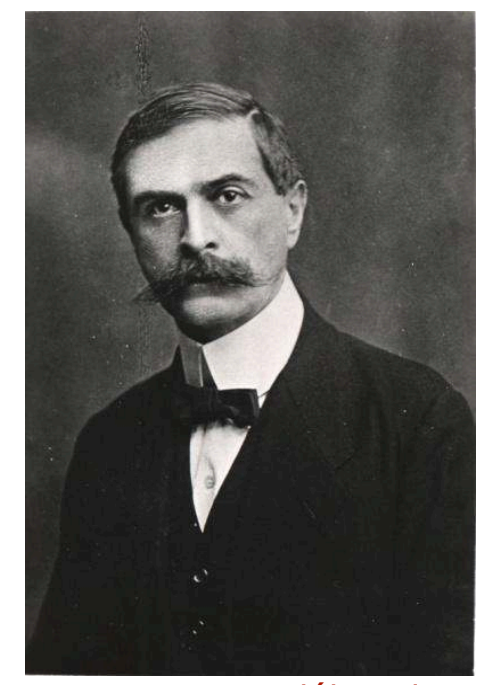

Figure 1: (en haut) Karl Landsteiner jeune, au début de ses années d'assistanat (vers 1896) ; (en bas) Billet de 1000 schillings autrichiens, de 1997 à 2001, avant passage à l'euro.

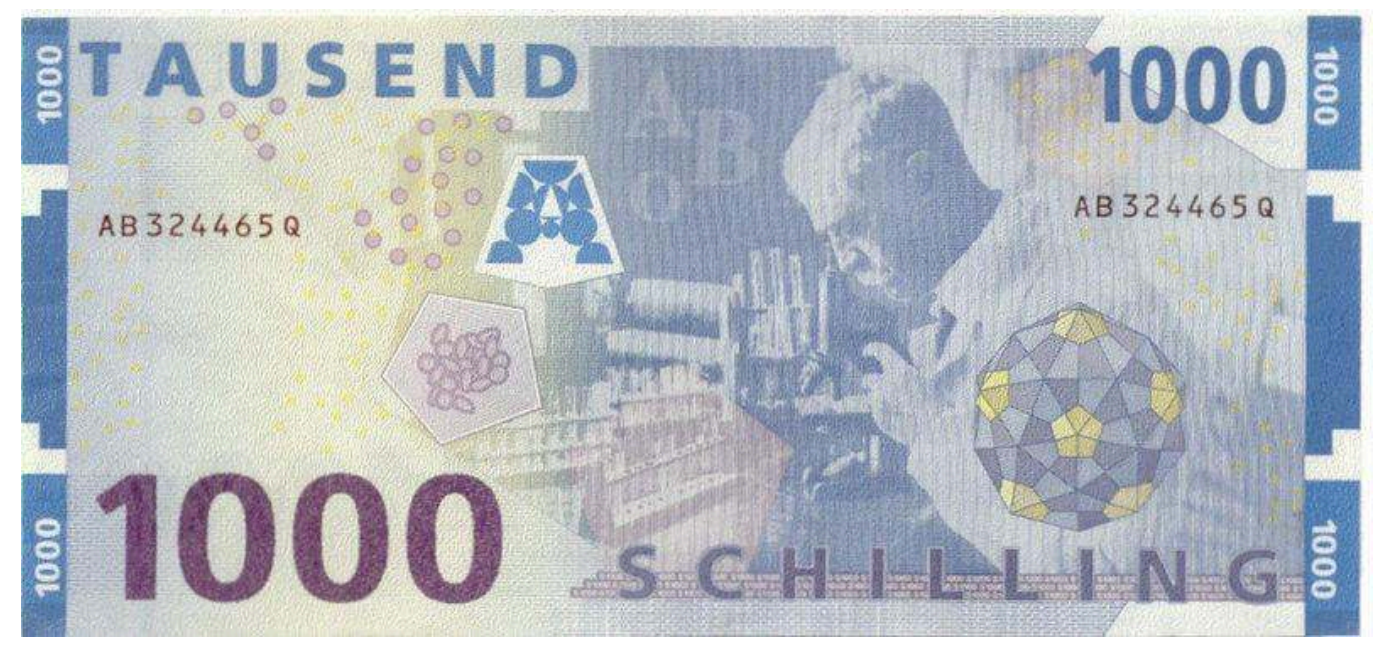




\section{DÉCOUVERTE DES GROUPES ABO (1900-1901)}

À l'orée du vingtième siècle, le monde de la recherche médicale se passionne pour l'immunologie naissante, particulièrement les mécanismes de défense antibactérienne; parmi les méthodes, encore rudimentaires, les techniques d'agglutination. C'est dans ce contexte qu'un jeune médecin de 33 ans, Karl Landsteiner, assistant d'Anton Weichselbaum à I'Institut d'anatomopathologie de I'université de Vienne, publie le texte fondateur de l'existence des groupes sanguins.

\section{Principe du groupage sanguin : I'agglutination des hématies ou hémagglutination}

Un groupe sanguin se définit par :

- la présence ou l'absence de certaines molécules antigéniques à la surface du globule rouge (ou hématie, ou érythrocyte) ;

- et, pour le système $A B O$ particulièrement, d'anticorps « naturels » anti-A et anti-B dans le plasma (ou dans le sérum, après coagulation).

Antigènes des globules rouges et anticorps plasmatiques sont mis en évidence par des réactions d'agglutination (sur plaque, sur microplaque, en tube, en gel de microfiltration) en présence de sérums-tests anti-A ou anti-B (épreuves globulaires) ou d'hématiestests $A$ ou $B$ (épreuves plasmatiques).

À titre d'exemple, une personne de groupe $\mathrm{O}$ n'a sur ses hématies ni antigène $A$, ni antigène $B$. Mais elle a dans son plasma des anticorps «naturels » anti-A et anti-B.

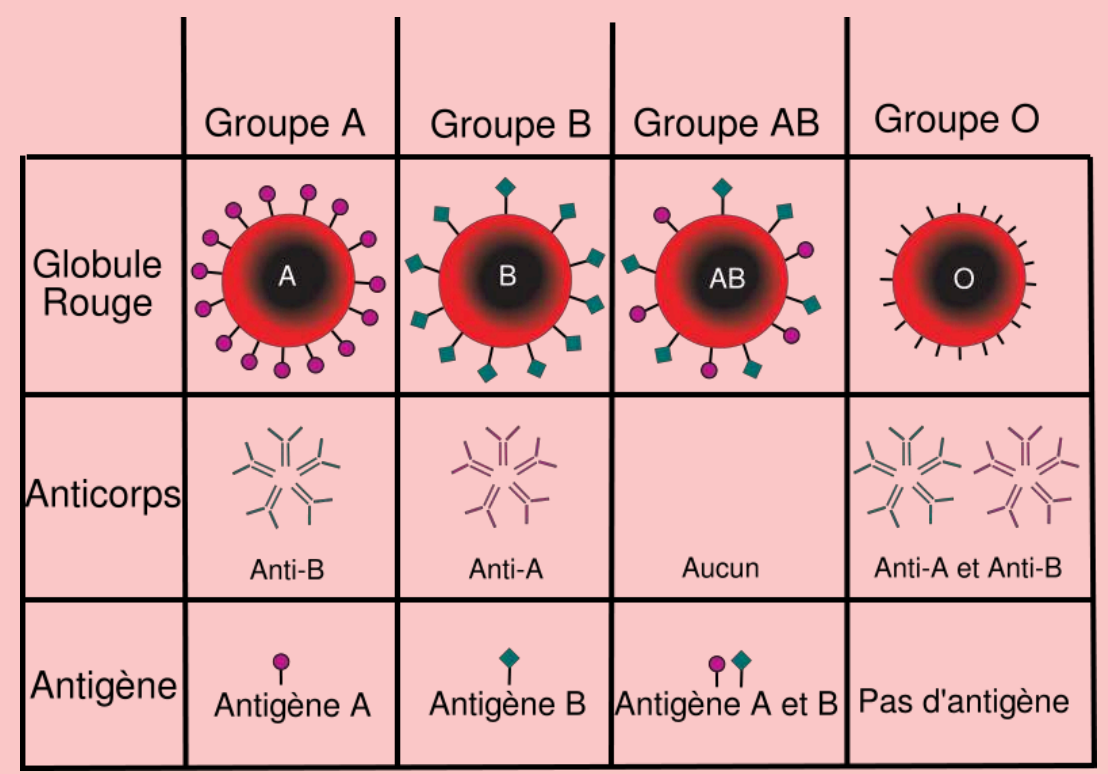

Figure 2 : Groupes sanguins du système ABO. Les hématies (globules rouges : $1^{e}$ ligne) sont mises en présence des sérums $\left(2^{e}\right.$ ligne). Le sérum $A$ contient des anticorps anti-B et agglutine donc les hématies de type $B$. Le sérum $O$ contient les deux anticorps anti- $A$ et anti- $B$ et agglutine toutes les hématies $A, B$ ou $A B$. 
Un anticorps est une molécule protéique, produite par le système immunitaire pour se fixer à une molécule étrangère, dite antigène (pour antibody generator). Ce mécanisme de lien antigène-anticorps est spécifique à chaque couple, de manière comparable à une clef et une serrure.

L'annonce de Landsteiner parut en deux temps: une brève mention dès 1900, suivie de l'article fondateur en 1901.

- Février 1900 : Landsteiner publie dans une revue de bactériologie un article sur les « effets antifermentatifs, lytiques et agglutinants du sérum et de la lymphe» (Centralblatt für Bakteriologie, Parasitenkunde und Infektionskrankheiten, 1900, 27, 357-62). Le texte est long, discursif, confus, mais, dans une note infra-paginale, on lit l'observation suivante :

Le sérum d'individus humains sains provoque l'agglutination non seulement des hématies animales mais aussi, souvent, des hématies d'autres individus. Il reste à déterminer si ce phénomène résulte de différences individuelles primitives ou de dommages, éventuellement d'origine bactérienne.

- Novembre 1901: I'article fondateur de la connaissance des groupes sanguins date du 14 novembre 1901, dans le N46 (volume 14, pages 11324) de la Wiener klinische Wochenschrift, sous le titre «Über Agglutinationserscheinungen normalen menschlichen Blutes ». Le texte est court, selon les critères de l'époque. La méthodologie est simple, fondée sur des réactions d'agglutination, mais l'article ne comporte aucune précision sur les modalités d'obtention des sérums ; pour les hématies, on sait seulement qu'elles proviennent d'une dilution de sang à $5 \%$ dans une solution de chlorure de sodium à $0,6 \%$. Sérum et suspension d'hématies sont mélangés à volume égal, en goutte ou en tube. Il n'y a pas de précision sur la température, l'intensité des réactions d'agglutination, le délai de lecture des résultats.

Les conclusions de Landsteiner découlent principalement des tableaux I et II, qui présentent les réactions d'agglutination entre sérums et hématies de six hommes sains, collaborateurs de l'institut d'anatomo-pathologie (tableau I : Dr Stoerk, Dr Pletschnig, Dr Sturli, Dr Erdheim, Dr Landsteiner, Mr Zaritsch laborantin), et de six accouchées (tableau II). Landsteiner identifie trois groupes, A, B et C, aux caractéristiques suivantes : 
- le sérum des sujets $A$ agglutine les hématies des sujets $B$, mais pas celles des autres sujets $A$;

- les hématies des sujets A sont agglutinées, de la même manière, par le sérum des sujets $B$;

- le sérum des sujets $C$ agglutine les hématies des sujets $A$ et $B$, mais pas celles des autres sujets $C$ (ce groupe $C$ sera rebaptisé $O$, quelques années plus tard, à l'initiative de Ludwik Hirszfeld et Emil von Dungern).

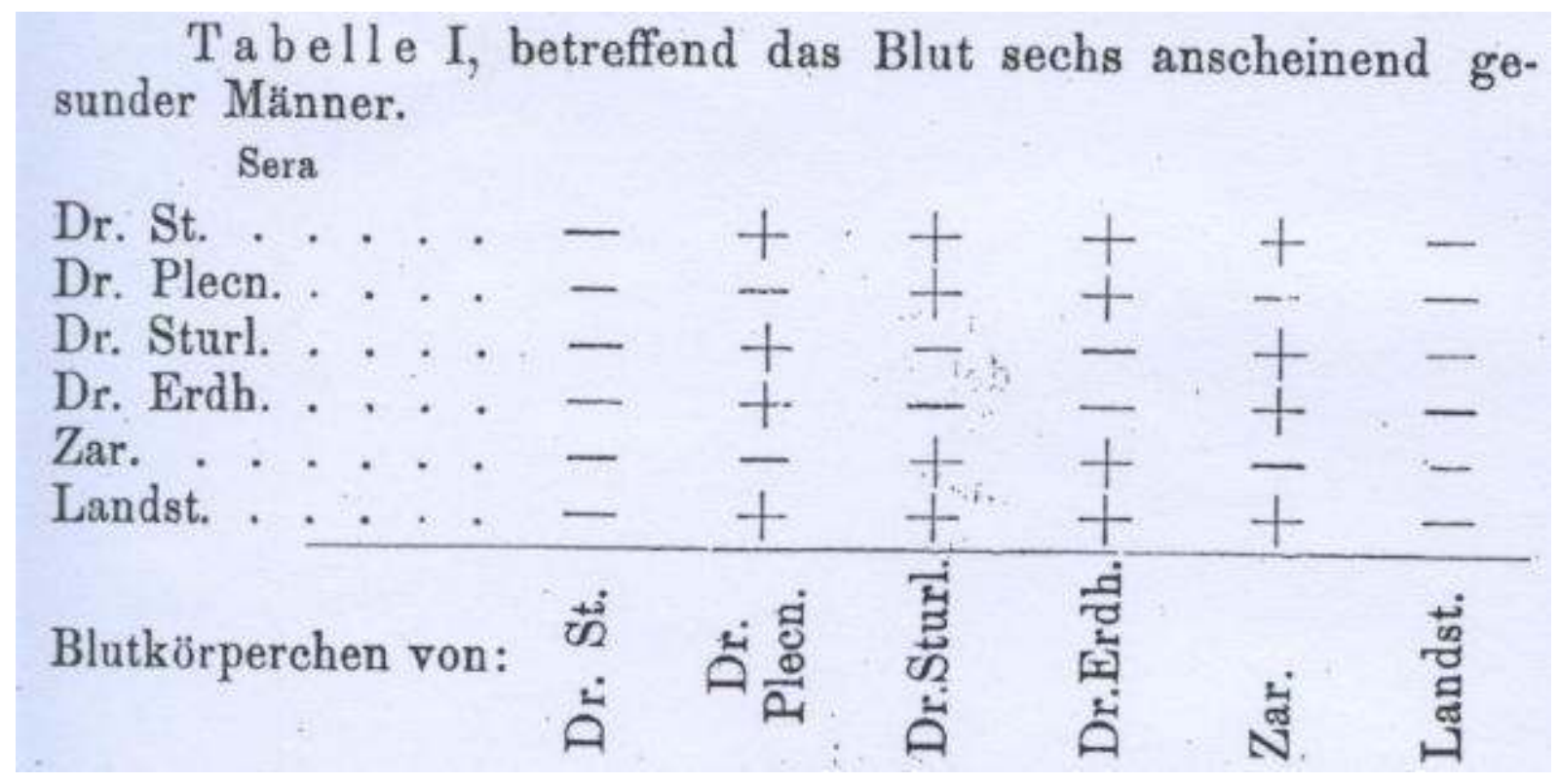

Figure 3 : Tableau I, page 1133, de I'article de Landsteiner. On constate que Landsteiner(Landst.) et Stoerk (Dr. St.) sont du même groupe; leurs sérums (lignes 1 et 6) agglutinent toutes les hématies sauf celles du même groupe : c'est le groupe O. Sturli (Dr. Sturl.) et Erdheim (Dr. Erdh.) sont du même groupe (identité de leurs lignes et colonnes) ; ce groupe ( $A$, ou $B$ ?) est différent de celui de Landsteiner et Stoerk ; leurs sérums n'agglutinent que les hématies des deux personnes restantes, Pletschnig (Dr. Plecn) et Zaritsch (Zar), qui appartiennent à un troisième groupe (B, ou $A$ ?).

L'inclusion de certains de ces cas dans des séries ultérieures plus importantes permet de supposer que, selon la terminologie actuelle, Adriano Sturli et Jakob Erdheim ${ }^{1}$ étaient très vraisemblablement du groupe $A$ et donc, Pletschnig et Zaritsch du groupe B (environ $45 \%$ de A et $9 \%$ de B dans la population « caucasienne »d'Europe occidentale).

Le tableau III n'est pas commenté. Il aurait pourtant permis d'intéressantes remarques puisqu'il présente les réactions d'agglutination entre les sérums de cinq des six accouchées du tableau II et six suspensions

1. Jakob Erdheim (1874-1937) devint professeur d'anatomo-pathologie à Vienne. C'est lui qui porta le diagnostic histologique de carcinome du palais lors de la première opération de Sigmund Freud en 1923. Son nom reste attaché à une maladie histiocytaire rare, la maladie d'Erdheim-Chester. 
d'hématies de sang de cordon (Nabelschnurblut), dont trois proviennent des nouveau-nés des accouchées ayant fourni les sérums.

Un quatrième tableau, concernant les réactions entre les sérums du tableau II (« et quelques autres »!) et les hématies du tableau I n'est pas même présenté, au prétexte que ces résultats sont «complètement concordants »! Enfin, Landsteiner se borne à mentionner, sans autre précision, les résultats identiques de l'étude de «dix autres personnes normales »:

Bei der Untersuchung von zehn anderen normalen Personen [...] fanden sich ähnliche Verhältnisse. [L'étude de dix autres personnes normales (...) donna des résultats identiques]

Dans la discussion, Landsteiner signale qu'une réaction d'agglutination a pu être obtenue avec un sérum desséché puis redissous, de même qu'avec du sang desséché : il suggère, en conséquence, l'intérêt potentiel de son travail en médecine légale. Enfin, ce n'est qu'aux dernières lignes de son article, et de manière laconique, presque désinvolte, que Landsteiner aborde le problème transfusionnel :

nos observations peuvent contribuer à expliquer les résultats variables des transfusions sanguines thérapeutiques chez l'homme.

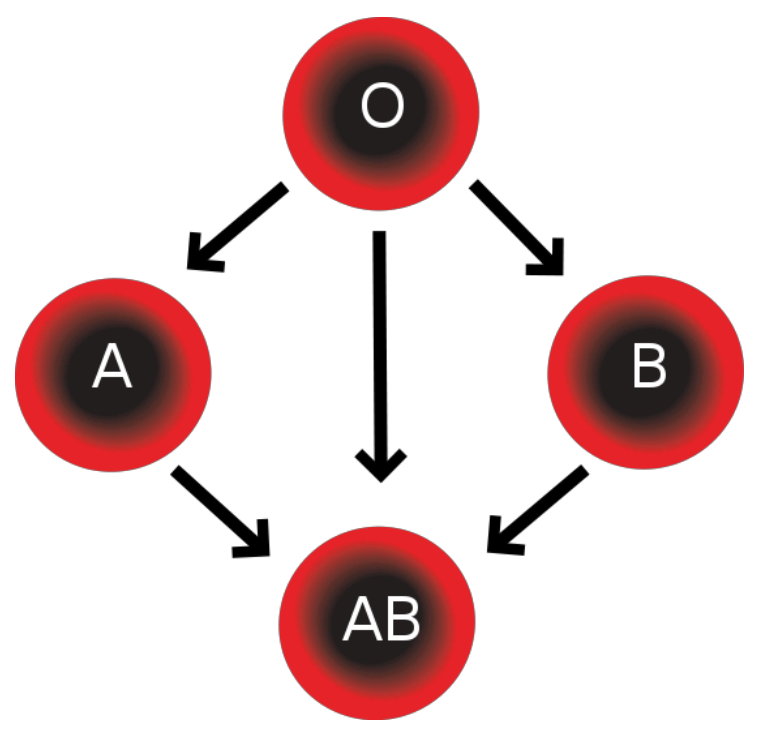

Figure 4 : Tableau de compatibilité en transfusion sanguine. En cohérence avec la figure 2, le sujet de groupe $O$ peut donner des hématies à tous les groupes (donneur universel) ; le sujet de groupe $A B$ peut recevoir des hématies de tous les groupes (receveur universel) - ceci indépendamment du système Rhésus. 


\section{KARL LANDSTEINER : UNE JEUNESSE VIENNOISE (1868-1901).}

Landsteiner naît le 14 juin 1868 à Baden, charmante petite ville au sud de Vienne, dans le vignoble, en lisière orientale de la forêt viennoise, où ses parents sont en villégiature. Il est le premier enfant, qui restera unique, de Léopold Landsteiner, journaliste et directeur de journal, et de son épouse née Franziska («Fanni ») Hess. Les époux Landsteiner appartiennent à la bourgeoisie juive aisée de Vienne, où ils habitent, quartier de Leopoldstadt.

Après de solides études secondaires au Maximilian-Staatsgymnasium, Karl Landsteiner entre à la faculté de médecine de I'Université de Vienne à l'automne $1885^{2}$. Le 21 février 1891, il est Docteur en Médecine (Doktor der gesamten Heilkunde). Sa formation post-doctorale est originale car elle comporte peu de stages cliniques et, au contraire, de longs séjours dans des laboratoires de chimie renommés, à Würzburg (Professeur Hermann Emil Fischer, Nobel de chimie en 1902), à Munich (Professeur Eugen Bamberger), à Zurich (Professeur Arthur Hantzsch). A l'évidence, Landsteiner se détourne très tôt de la médecine clinique, qu'il n'exercera jamais, et exprime son attrait pour une carrière de chercheur en biologie humaine, qu'il entend mener avec les outils et les méthodes de la chimie.

En janvier 1896, à 27 ans, Landsteiner entre comme assistant à l'institut d'hygiène de I'Université de Vienne, dirigé par le professeur Max Gruber (18531927). Un des thèmes de recherche de l'institut est alors le «phénomène de Pfeiffer $^{3} \gg$. Gruber et I'un de ses élèves, I'anglais Herbert Edward Durham (18661945), s'efforcent de reproduire in vitro le phénomène de Pfeiffer : en présence

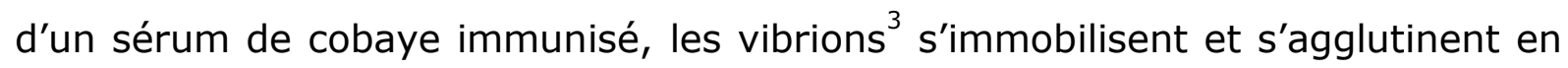
amas. Gruber et Durham étendent ensuite leurs recherches au pouvoir agglutinant du sérum humain sur diverses bactéries, dont le bacille typhique (Salmonella typhi ${ }^{4}$ ).

2. En juillet 1885, Landsteiner termine ses études secondaires par un succès aux épreuves de la Maturitätsprüfung (familièrement appelée «Matura »), équivalent de notre baccalauréat. Les appréciations du jury sont particulièrement élogieuses en sciences naturelles et mathématiques.

3. Bactériologiste allemand, élève de Robert Koch, Richard Pfeiffer (1858-1945) étudie dans les années 18941895 I'infection expérimentale du cobaye par le vibrion cholérique (Vibrio cholerae : bactérie Gram négatif, en forme de bâtonnet incurvé, munie d'un flagelle qui lui donne sa mobilité ; c'est l'agent du choléra). Après injection intra-péritonéale d'une culture de vibrion à un cobaye, il constate la mobilité des germes et leur multiplication jusqu'à la mort de l'animal. Par contre, la même injection à un cobaye déjà immunisé contre le choléra n'est pas mortelle: les vibrions perdent leur mobilité, prennent une forme arrondie, pâlissent rapidement et disparaissent du liquide péritonéal. C'est le «phénomène de Pfeiffer ».

4. Bacille de la fièvre typhoïde, découvert en 1880 par Karl Joseph Eberth (1835-1926), qui lui donne le nom de Bacillus typhosus. 


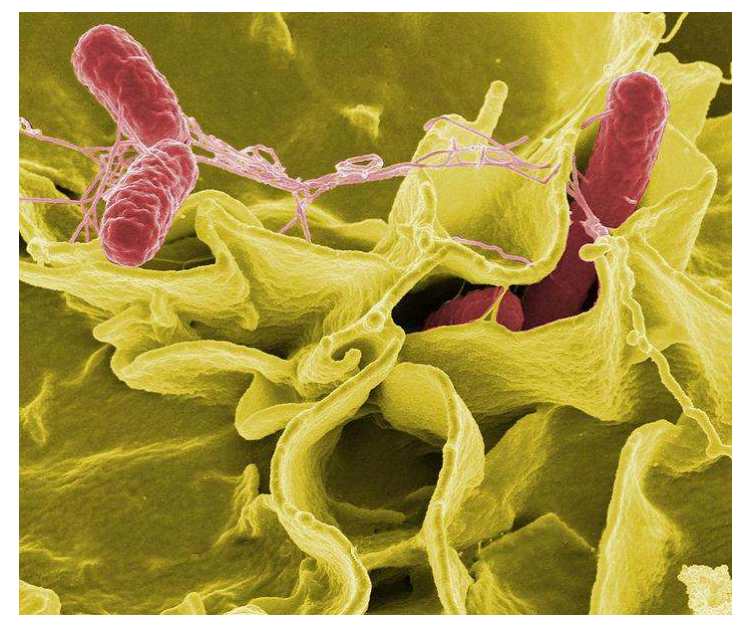

Figure 5 : Salmonella typhimurium (en rose) sur une culture de cellules humaines (image Wikimedia Commons - origine NIH). Cette bactérie provenant des rongeurs se transmet à l'homme par ingestion d'eaux usées. C'est un des nombreux agents des entérites à salmonelles.

Landsteiner est associé à ces travaux. Il montre d'abord que l'agglutination bactérienne par un sérum immun n'est que partiellement spécifique : dans le cas d'un sérum de cobaye immunisé contre Bacillus typhimurium, l'agglutination est complète pour cette bactérie mais existe aussi, incomplète, pour des souches ou des espèces bactériennes voisines. Puis il évalue la survie de cobayes infectés par injection intra-péritonéale de Bacillus typhimurium en quantités croissantes: la durée de survie est d'autant plus longue que la charge bactérienne injectée est faible ; surtout, la survie des animaux est particulièrement longue si du sérum immun de cobaye est ajouté à l'injection bactérienne intra-péritonéale. Enfin, sur un modèle expérimental de culture in vitro, Landsteiner analyse la cinétique de prolifération bactérienne en fonction de divers paramètres, comme l'apport de sérum de cobaye immun ou non-immun et l'apport d'exsudat péritonéal de cobaye, utilisé à l'état frais ou modifié par centrifugation ou congélation (Wiener klinische Wochenschrift, 1897, 10, 439-44). Dans ce long travail expérimental sur les défenses immunologiques antibactériennes, Landsteiner est encore très loin de la mise en évidence des groupes sanguins !

En 1897, il quitte I'Institut d'hygiène pour I'Institut d'anatomo-pathologie que dirige Anton Weichselbaum (1845-1920). Dans son travail de recherche, il s'inspire toujours du modèle expérimental de Pfeiffer mais s'écarte du champ de la bactériologie. Il injecte dans la cavité péritonéale de lapins du sang défibriné de cobaye, de chien ou de cheval et observe que le pouvoir d'hémolyse ou d'agglutination du sérum de ces lapins augmente nettement en comparaison de celui d'animaux témoins. De plus, ces modifications sont spécifiques d'espèce : 
ainsi, l'augmentation du pouvoir agglutinant du sérum de lapin recevant du sang de chien est particulièrement nette avec les hématies de chien (Centralblatt für Bakteriologie, Parasitenkunde und Infektionskrankheiten, 1899, 25, 546-9). Dans la carrière de Landsteiner, cette étude peut sembler marginale; elle préfigure pourtant toute l'œuvre à venir sur les groupes sanguins et l'immunologie érythrocytaire.

Ainsi, les conclusions présentées dans l'article fondateur de 1901 apparaissent-elles comme l'aboutissement d'une lente progression intellectuelle et expérimentale, faite de glissements successifs : glissements méthodologiques depuis l'agglutination des bactéries jusqu'à l'agglutination d'hématies animales puis d'hématies humaines, glissements conceptuels depuis un phénomène pathologique, d'origine infectieuse, jusqu'à un phénomène physiologique. La découverte des groupes sanguins $A B O$ apparaît donc comme la conséquence, longuement mûrie entre 1896 et 1901, des travaux confiés par Gruber au jeune Landsteiner sur l'analyse du « phénomène de Pfeiffer ».

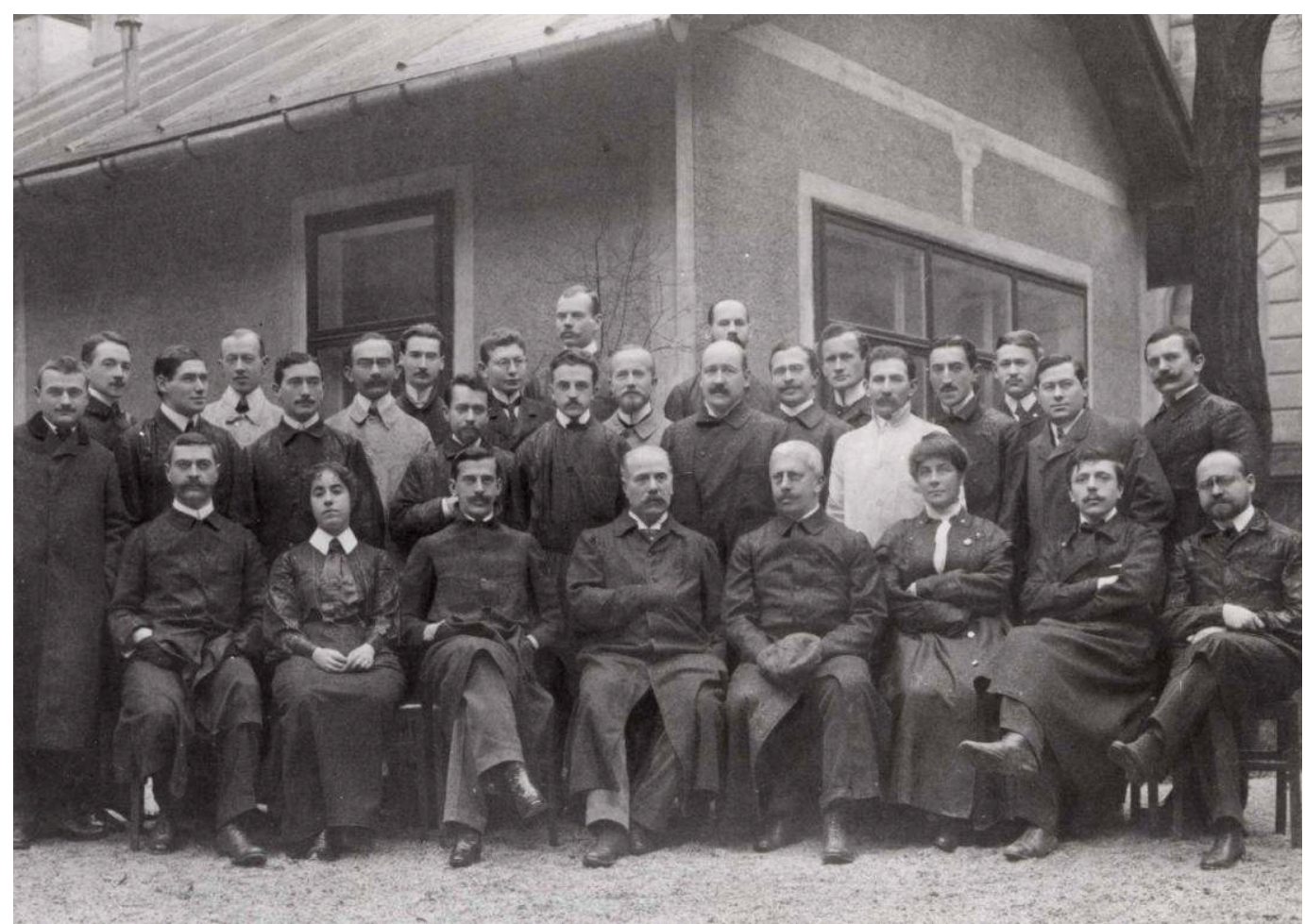

Figure 6 : Anton Weichselbaum, assis au centre, et son équipe de l'institut d'anatomo-pathologie, en 1905. On reconnaît Karl Landsteiner, assis à l'extrême gauche, et Oskar Stoerk, assis à la droite de Weichselbaum. 


\section{APRÈs LA DÉCOUVERTE DES GROUPES ABO (1901-1943).}

Landsteiner reste à I'Institut d'anatomo-pathologie jusqu'en décembre 1907. Fort de sa solide formation de chimiste, il conçoit très vite l'agglutination des hématies comme une réaction chimique dont il analyse méthodiquement les facteurs de variations tels que la température, la concentration en hématies, l'âge des sujets testés ${ }^{5}$. Il observe que les agglutinines «immunes » sont plus résistantes à la chaleur que les agglutinines «normales», première avancée vers la distinction maintenant classique entre anticorps anti-érythrocytaires immuns et naturels.

\section{Les anticorps du système $\mathrm{ABO}$}

Comme cela apparaît dans la Figure 2, le système ABO est original par la présence constante, dans le plasma (et le sérum), d'anticorps correspondant aux antigènes absents sur les hématies. Ces anticorps sont appelés « réguliers » ou «naturels ». Leur concentration sérique est très faible à la naissance et augmente progressivement pendant I'enfance.

Ces anticorps «naturels » ont des caractéristiques physico-chimiques différentes de celles des anticorps «immuns » ou «irréguliers 》. Ceuxci apparaissent ponctuellement, en conséquence d'un épisode d'immunisation, habituellement transfusionnelle ou fœto-maternelle.

Grâce au travail d'Adriano Sturli (1873-1966) et Alfred DecastelloRechtwehr (1872-1960) (Münchener medicinische Wochenschrift, 1902, 49, 1090-5), jeunes collaborateurs occasionnels de Landsteiner à I'Institut d'anatomo-pathologie, s'impose progressivement l'existence d'un quatrième groupe, $A B$, à côté des groupes $A, B$ et $C$.

En décembre 1907, Landsteiner quitte l'institut d'anatomo-pathologie et prend la direction, avec le titre de prosecteur ${ }^{6}$, du service d'anatomo-pathologie de l'hôpital Wilhelmine (Wilhelminenspital) à Ottakring, dans I'ouest de Vienne. Il est nommé professeur adjoint d'anatomo-pathologie en 1911. En 1916, à l'âge de 48 ans, il épouse Léopoldine Hélène Wlasto, actrice de théâtre de 36 ans, d'origine grecque par son père, un des responsables laïcs de la paroisse

5. Dans un travail en collaboration avec le gynécologue-obstétricien Josef Halban (1870-1937), Landsteiner fait une analyse comparative du pouvoir agglutinant et hémolysant du sérum des mères et de leurs enfants nouveau-nés. Il conçoit alors clairement la notion d'immaturité immunologique néonatale : « ... l'organisme du nouveau-né, comparé à celui de l'adulte, doit être considéré comme incomplètement développé. » (Münchener medicinische Wochenschrift, 1902, 49, 473-6). Cette notion est de nos jours unanimement admise puisque, chez l'enfant, un groupe sanguin « définitif » ne peut être établi avant l'âge de 6 mois.

6. Dans le monde médical germanique, le mot prosecteur (Prosektor) désigne le médecin-chef d'une « prosecture » (Prosektur), c'est-à-dire du service d'anatomo-pathologie d'un hôpital. 
orthodoxe grecque de Vienne. Leur fils Ernst, qui sera leur unique enfant, naît en 1917. En janvier 1920, chassée par la lourde misère qui écrase la petite Autriche après l'effondrement de l'Empire, la famille quitte Vienne pour la Hollande, où Landsteiner prend un poste de prosecteur à I'hôpital de la Croix Rouge de La Haye. Cet intermède hollandais sera bref. Landsteiner est sollicité par Simon Flexner (1863-1946), directeur de I'Institut Rockefeller et, en avril 1923, quitte La Haye pour New York : il occupe un poste de chercheur à l'Institut Rockefeller, jusqu'à sa retraite en 1939 et même, bénévolement, jusqu'à sa mort en 1943.

Dans son laboratoire de l'institut Rockefeller, Landsteiner peut enfin se consacrer à plein temps à sa passion, la recherche. Il poursuit son exploration des groupes $\mathrm{ABO}$ :

- Avec son assistant Charles Philip Miller (1894-1985), il met en évidence la présence des antigènes des groupes $A B O$ sur les hématies des grands singes anthropoïdes.

- Avec Philip Levine (1900-1987), un autre de ses assistants, il confirme la subdivision du groupe $A$ en sous-groupes $A_{1}$ et $A_{2}{ }^{7}$. En outre, il montre le caractère probablement héréditaire de la transmission de ces sous-groupes, ainsi que leur répartition différente entre personnes de races noire et blanche.

- Dans un article de 1926, Landsteiner et Levine rapportent la présence de substances $A$ et $B$ sur les spermatozoïdes humains, selon la même distribution que pour les hématies ${ }^{8}$. Ce résultat peut apparaître anecdotique; il est important, néanmoins, comme un des premiers arguments en faveur du caractère ubiquitaire des antigènes du système $A B O$.

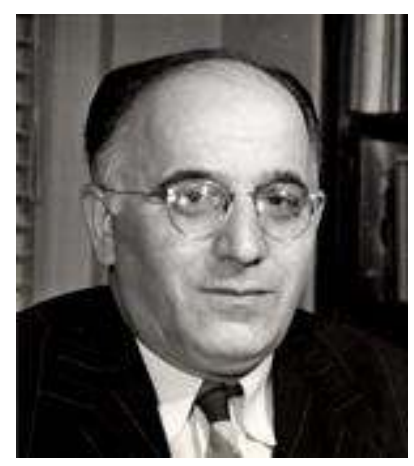

Fiqure 7 : Philip Levine, assistant de Landsteiner à l'institut Rockefeller de 1925 à 1932.

7. Dans le sous-groupe A1 ( $80 \%$ environ des sujets A), les molécules antigéniques A sont plus abondantes (environ 1000000 par hématie) et mieux exposées à la surface de la cellule que dans le sous-groupe A2 (environ 400000 molécules par hématie).

8. "The sperm cells therefore contain substances identical or similar to the isoagglutinable factors $A$ and $B$ of human red blood cells." (Journal of Immunology, 1926, 12, 415-8). 
Après 1901, 1927 est une autre année faste dans I'histoire des groupes sanguins. Cette année-là, Landsteiner et Levine découvrent sur les hématies humaines, deux « facteurs agglutinants » qu'ils désignent par les lettres $M$ et $N$ : ils s'avèrent indépendants des groupes $A B O$ et constituent les premiers antigènes connus d'un ensemble complexe, le système MNSs, dont la connaissance sera complétée vers $1950^{9}$. Toujours en 1927, Landsteiner et Levine découvrent I'antigène $P$, lui aussi indépendant des groupes $A B O$ (actuellement: système $\mathrm{P}_{1} \mathrm{PK} ; \mathrm{N}^{\circ} 003$ de la classification ISBT).

Le 21 juin 1929, Karl Landsteiner, son épouse Hélène et leur fils Ernst, alors respectivement âgés de 61,48 et 12 ans deviennent citoyens des ÉtatsUnis d'Amérique. En 1930, Landsteiner est lauréat du prix Nobel de Physiologie ou Médecine pour «sa découverte des groupes sanguins humains». À Stockholm, il prononce la traditionnelle conférence des lauréats, en allemand, sur le thème des «Différences individuelles du sang humain» (« Über individuelle Unterschiede des menschlichen Blutes »).

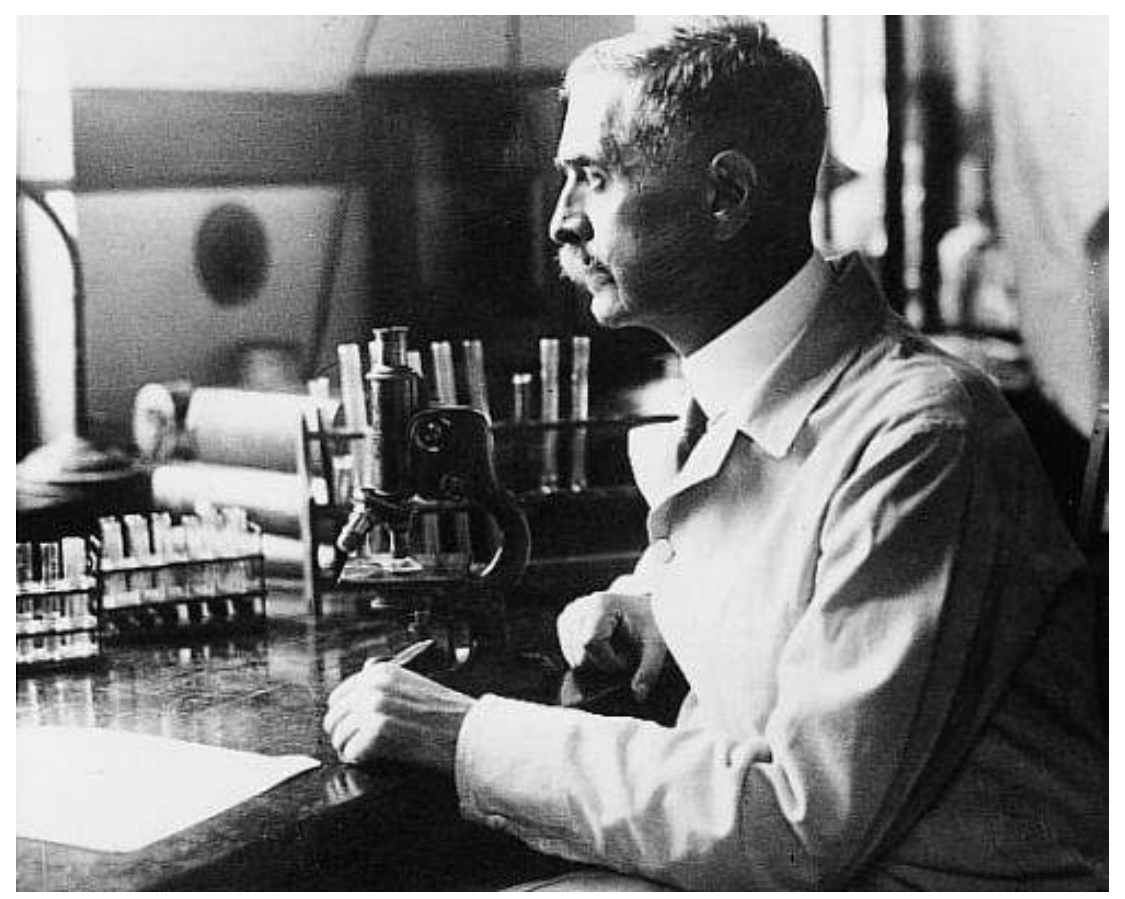

Figure 8 : Landsteiner dans son laboratoire de l'institut Rockefeller, à 62 ans, à I'époque du prix Nobel (c'est dans ce même environnement qu'il apparaît dans le billet de 1000 shillings, Figure 1).

L'ultime contribution majeure de Landsteiner à la connaissance des groupes sanguins concerne le «facteur rhésus». Pourtant, la mise en évidence de ce

9. Ce système de groupes sanguins est actuellement dénommé MNS et porte le $N^{\circ} 002$ de la classification ISBT (International Society of Blood Transfusion). 
facteur revient à Philip Levine, dès 1937, avec la description de l'anticorps correspondant chez une femme ayant récemment accouché d'un fœetus mort (Journal of the American Medical Association, 1939, 113, 126-7). Un peu plus tard, en 1940-1941, Landsteiner et Alexander Wiener (1907-1976) retrouvent ce facteur par un modèle expérimental d'hétéro-immunisation de lapin par des hématies de singe Macacus rhesus, d'ou le nom de «facteur rhésus » (Journal of experimental Medicine, $1941, \mathbf{7 4}, 309-20)^{10}$.

Le 24 juin 1943, dans son laboratoire, Landsteiner est pris d'une violente douleur thoracique, très évocatrice d'un angor aigu. Il meurt le 26 juin 1943 à I'hôpital de l'institut Rockefeller. Son corps est incinéré, ses cendres enterrées dans l'île de Nantucket, au large de la Nouvelle Angleterre.

\section{ET MAINTENANT (2012)?}

Le monde des groupes sanguins est devenu un ensemble complexe de 30 systèmes, comportant quelque 280 antigènes, 6 collections et 2 séries, d'antigènes de grande fréquence (série 901 , ou «antigènes publics») et de faible fréquence (série 700 , ou «antigènes privés »). Pour ces molécules antigéniques, la masse des connaissances acquises est impressionnante, concernant leur génétique et génomique, leur structure biochimique et leur répartition, leur pouvoir immunogène, leur fonction physiologique. Quel immense chemin parcouru depuis I'article de 1901 par Karl Landsteiner, alors jeune assistant d'Anton Weichselbaum ! Les quelque trois millions de produits sanguins transfusés annuellement, en France, à plus de 550000 patients, témoignent de façon éclatante de la puissance fondatrice de ce texte.

(juillet 2012)

10. Dans la découverte du «facteur rhésus », les rôles moteurs sont tenus par Philip Levine puis, un peu plus tard, Alexander Wiener. Le rôle de Landsteiner a surtout été de favoriser et couvrir de son autorité scientifique les recherches de Wiener. Le «facteur rhésus » est maintenant connu sous le nom d'antigène $D$, ou $R H_{1} d u$ système Rhésus (système $\mathrm{Rh}, \mathrm{N}^{\circ} 004$ de la classification ISBT). Landsteiner ne prit qu'une part infime à I'analyse des complexités immunologiques, biochimiques et génétiques de ce système. 